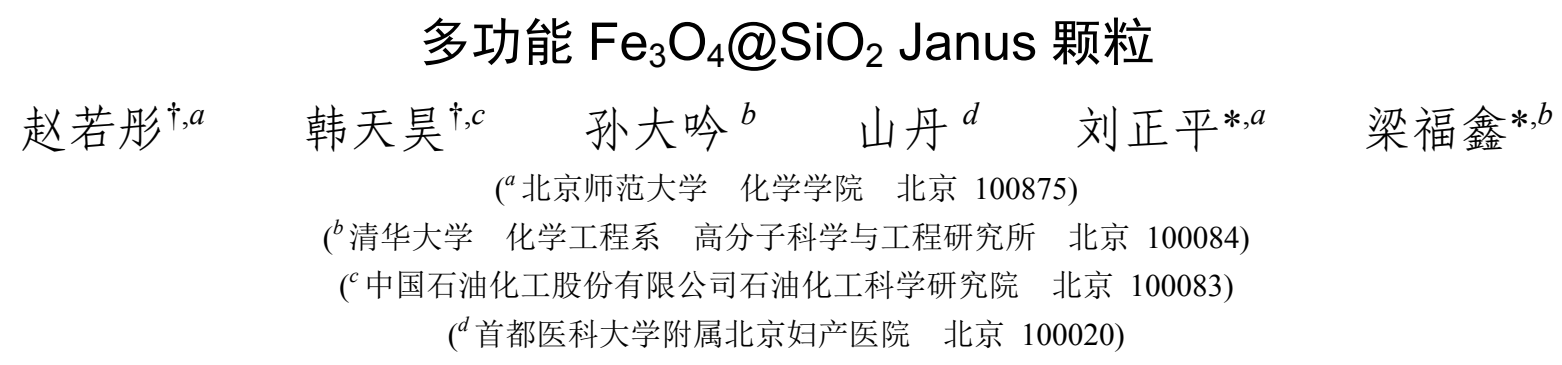

摘要 采用溶剂热法和溶胶-凝胶法制备了顺磁性 $\mathrm{Fe}_{3} \mathrm{O}_{4} @ \mathrm{SiO}_{2}$ 颗粒, 以 Pickering 乳液界面保护法实现颗粒表面分区获 得 $\mathrm{Fe}_{3} \mathrm{O}_{4} @ \mathrm{SiO}_{2} \mathrm{Janus}$ 颗粒, 进一步选区复合生长 $\mathrm{Pt}$ 或 $\mathrm{Ag}$ 纳米颗粒制备 $\mathrm{Fe}_{3} \mathrm{O}_{4} @ \mathrm{SiO}_{2}-\mathrm{Pt}$ 和 $\mathrm{Fe}_{3} \mathrm{O}_{4} @ \mathrm{SiO}_{2}-\mathrm{Ag} \mathrm{Janus}$ 颗粒. $\mathrm{Fe}_{3} \mathrm{O}_{4} @ \mathrm{SiO}_{2}-\mathrm{Pt}$ Janus 颗粒的 $\mathrm{Pt}$ 一侧进行催化过氧化氢的反应, 具有自驱动功能. 因其顺磁性和两亲性, $\mathrm{Fe}_{3} \mathrm{O}_{4} @ \mathrm{SiO}_{2}-\mathrm{Ag}$ Janus 颗粒能够作为磁响应颗粒乳化剂稳定油水乳液, 并将 $\mathrm{Ag}$ 的催化功能引入界面.

关键词 Janus 颗粒; 磁响应; 自驱动; 固体乳化剂; 催化

\title{
Multifunctional $\mathrm{Fe}_{3} \mathrm{O}_{4} @ \mathrm{SiO}_{2}$ Janus Particles
Zhao, Ruotong $^{\dagger, a} \quad$ Han, Tianhao $^{\dagger, c} \quad$ Sun, Dayin $^{b} \quad$ Shan, Dan $^{d} \quad$ Liu, Zhengping*,a Liang, Fuxin* ${ }^{* b}$
$\left({ }^{a}\right.$ College of Chemistry, Beijing Normal University, Beijing 100875) ( ${ }^{c}$ Sinopec Research Institute of Petroleum Processing, China Petroleum and Chemical Corporation, Beijing 100083)
( ${ }^{d}$ Beijing Obstetrics and Gynecology Hospital, Capital Medical University, Beijing 100020) \\ ( ${ }^{b}$ Institute of Polymer Science and Engineering, Department of Chemical Engineering, Tsinghua University, Beijing 100084)
}

\begin{abstract}
Fe}_{3} \mathrm{O}_{4} @ \mathrm{SiO}_{2}$ particles were synthesized by a solvothermal method and a classical stöber method. Superparamagnetic $\mathrm{Fe}_{3} \mathrm{O}_{4}$ was the core, and a sol-gel coating of $\mathrm{SiO}_{2}$ was the shell. After the $\mathrm{SiO}_{2}$ surface was modified with amino groups, benzaldehyde was conjugated to the particles by a Schiff base reaction. The $\mathrm{Fe}_{3} \mathrm{O}_{4} @ \mathrm{SiO}_{2}$ particles were emulsified in paraffin/water as a solid emulsifier to obtain an oil-in-water Pickering emulsion. After cooling the paraffin, the particles were fixed on the surface of the emulsion droplets. The particles were etched in ammonium fluoride aqueous solution, and Janus particles with different structures could be obtained by adjusting the etching time. Via the in situ growth of metal Pt or Ag nanoparticles, superparamagnetic $\mathrm{Fe}_{3} \mathrm{O}_{4} @ \mathrm{SiO}_{2}-\mathrm{Pt}$ or $\mathrm{Fe}_{3} \mathrm{O}_{4} @ \mathrm{SiO}_{2}-\mathrm{Ag}$ Janus particles were obtained. The movement of $\mathrm{Fe}_{3} \mathrm{O}_{4} @ \mathrm{SiO}_{2}-\mathrm{Pt}$ Janus particles was observed due to the catalytic decomposition of hydrogen peroxide aqueous solution. It was found that in the short term, there was a linear motion, while in the long term, the motion direction and trajectory were random. $\mathrm{Fe}_{3} \mathrm{O}_{4} @ \mathrm{SiO}_{2}-\mathrm{Ag}$ Janus particles were used as magnetic solid surfactants to stabilize the emulsions and catalyze the nitro reduction. About $60 \%$ of the surficial area of the Janus particles was modified by phenyl groups, while the remaining $40 \%$ was covered with Ag nanoparticles. Under the premise of maintaining the Janus balance, the whole particle became more hydrophobic, which was conducive to the formation of the water-in-oil emulsion. In addition, the Ag side of the Janus particles was towards the aqueous phase, and the opposite hydrophobic side was towards the oil phase. The Janus particles possessed a fixed orientation assembly at the oil-water interface. The assemble membrane possessed Janus characteristics, and it facilitated the stable dispersion of the emulsion and the catalysis. The method has the advantages of a simple principle, capability for mass production, universality and versatility. It is expected that Janus particles will be used to more precisely regulate the zoning with different functional substances.
\end{abstract}

Keywords Janus particle; magnetic response; self-driven; solid emulsifier; catalysis

\section{1 引言}

Janus 颗粒表面具有不同化学或功能分区，能够同 时赋予颗粒两种不同、甚至相反的性质，如亲水/疏水、
阴离子/阳离子、极性/非极性 ${ }^{[1]}$. Janus 颗粒独特的化学组 成及结构使其拥有许多独特的性能, 在模拟两亲性分子 组装 ${ }^{[2]}$, 固体乳化剂 ${ }^{[3-7]}$, 界面催化 ${ }^{[6,7]}$, 显示 ${ }^{[8,9]}$, 光学材 料 $^{[10-12]}$, 自驱动马达 ${ }^{[13-16]}$, 特殊浸润性光子晶体膜 ${ }^{[17]}$,

\footnotetext{
*E-mail: lzp@bnu.edu.cn; liangfuxin@tsinghua.edu.cn

$\uparrow$ These authors contributed equally to this work

Received June 4, 2020; published July 13, 2020.

Supporting information for this article is available free of charge via the Internet at http://sioc-journal.cn.

Project supported by the National Natural Science Foundation of China (Nos. 51673119, 51622308).

项目受国家自然科学基金(Nos. 51673119, 51622308)资助.
} 
LB 膜 ${ }^{[18]}$, 生物方向 ${ }^{[19]}$ 等领域展示了诱人的应用前景. 例如，一侧亲水、另一侧亲油的 Janus 颗粒具有类似于 小分子表面活性剂或嵌段共聚物的双亲性, 能够作为固 体乳化剂稳定乳液 ${ }^{[20-24]}$. Janus 颗粒与传统的均质颗粒相 比更容易吸附在两相界面 ${ }^{[25-28]}$. Binks 等发现 Janus 颗粒 在油水界面的脱附能要比均质颗粒高出 3 倍, 即使当 Janus 颗粒与界面的平均接触角为 $0^{\circ}$ 和 $180^{\circ}$ 时, 颗粒依 然可以保持很强的吸附能力, 这是均质颗粒难以实现 的 ${ }^{[26,29,30]}$. 同时, 凭借颗粒的 Pickering 效应, Janus 颗粒 能够优先分布在界面并保持稳定 ${ }^{[25]}$.

通过表面化学分区进一步复合功能组成, Janus 颗 粒能够衍生诸多功能 ${ }^{[5,31]}$. 在 Janus 颗粒一侧复合金属颗 粒, 并通过化学反应产生气体, 利用这种独特的不对称 结构实现自驱动. 例如, 由 $\mathrm{Au}-\mathrm{Pt}$ 或 $\mathrm{Au}-\mathrm{Ni}$ 组成棒状双 金属 Janus 颗粒, 与过氧化氢 $\left(\mathrm{H}_{2} \mathrm{O}_{2}\right)$ 反应产生气体获得 驱动能 ${ }^{[32-36]}$. $\mathrm{Ge}$ 等 ${ }^{[37]}$ 介绍了通过光还原法制备了不对 称的 $\mathrm{TiO}_{2} / \mathrm{MnO}_{2}$ 纳米机器, 是靠气体推进获得驱动力. Zheng 等 ${ }^{[38]}$ 制备了光驱动的 $\mathrm{Zn}_{x} \mathrm{Cd}_{1-x} \mathrm{Se}-\mathrm{Cu}_{2} \mathrm{Se}-\mathrm{Pt}$ 纳米线. $\mathrm{He}$ 等 ${ }^{[39]}$ 制备了 $\mathrm{Au}$ 纳米颗粒修饰的介孔二氧化硅 Janus 颗粒, 颗粒表面在近红外下会产生热梯度, 使颗粒实现 高速运动. $\mathrm{He}$ 等 ${ }^{[40]}$ 进一步合成了铂纳米颗粒包覆的多 层中空胶囊, 不仅可以实现药物释放, 也可通过催化过 氧化氢分解的氧气气泡实现自主移动. Resasco 等 ${ }^{[41]}$ 将 Pd 沉积在碳纳米管的亲水性二氧化硅上制备了 Janus 催 化剂, 发现 Janus 颗粒作为催化剂可以稳定水油乳液并 在界面实现催化反应. Kirillova 等 ${ }^{[42]}$ 首次报道了用聚苯 乙烯(PS)和聚丙烯酸(PAA)制备具有毛发状的 Janus 型 催化剂, 复合的 $\mathrm{Ag}$ 或 $\mathrm{Au}$ 纳米粒子透过 PAA 侧催化降 解 4-硝基苯酚、曙红 Y 和亚甲基蓝等染料. Wang 等 ${ }^{[43]}$ 报道了一种亚铃状的金属-金属氧化物的纳米粒子, 能 够催化 $\mathrm{CO}$ 氧化.

本工作中, 我们通过 Pickering 乳液方法制备顺磁 性 $\mathrm{Fe}_{3} \mathrm{O}_{4} @ \mathrm{SiO}_{2}$ Janus 颗粒, 并通过分别复合 $\mathrm{Pt}$ 和 $\mathrm{Ag}$ 纳 米颗粒得到 $\mathrm{Fe}_{3} \mathrm{O}_{4} @ \mathrm{SiO}_{2}-\mathrm{Pt}$ 和 $\mathrm{Fe}_{3} \mathrm{O}_{4} @ \mathrm{SiO}_{2}-\mathrm{Ag} \mathrm{Janus}$ 颗 粒, 赋予 Janus 颗粒自驱动和催化功能. 如图 1 所示, 通 过氨基与醛基形成的席夫碱动态键, 使 $\mathrm{Fe}_{3} \mathrm{O}_{4} @ \mathrm{SiO}_{2}$ 颗 粒表面修饰有苯甲醛基团, 用改性后的 $\mathrm{Fe}_{3} \mathrm{O}_{4} @ \mathrm{SiO}_{2}$ 颗 粒形成水包石蜡的 Pickering 乳液, 颗粒在石蜡微球表 面形成单层分布, 其一侧嵌入凝固的石蜡中, 通过氟化 铵 $\left(\mathrm{NH}_{4} \mathrm{~F}\right)$ 水溶液蚀刻朝向水相的一侧, 则可获得暴露的 硅羟基. (1)未被刻蚀的一侧有残余的氨基, 可进一步复 合 Pt 纳米颗粒, 硅着基一侧修饰聚环氧乙烷(PEO), 获 得 $\mathrm{Fe}_{3} \mathrm{O}_{4} @ \mathrm{SiO}_{2}$-Pt Janus 颗粒; (2)未被刻蚀的一侧有残余 的氨基, 可进一步复合 $\mathrm{Ag}$ 纳米颗粒, 硅羟基一侧修饰 苯基, 获得两亲性 $\mathrm{Fe}_{3} \mathrm{O}_{4} @ \mathrm{SiO}_{2}-\mathrm{Ag}$ Janus 颗粒. $\mathrm{Fe}_{3} \mathrm{O}_{4} @ \mathrm{SiO}_{2}-\mathrm{Pt}$ Janus 颗粒通过 $\mathrm{Pt}$ 催化 $\mathrm{H}_{2} \mathrm{O}_{2}$ 产生 $\mathrm{O}_{2}$ 实 现自驱动, 两亲性 $\mathrm{Fe}_{3} \mathrm{O}_{4} @ \mathrm{SiO}_{2}-\mathrm{Ag}$ Janus 颗粒作为固体 乳化剂将 $\mathrm{Ag}$ 纳米颗粒引入油水乳液界面实现催化作用.

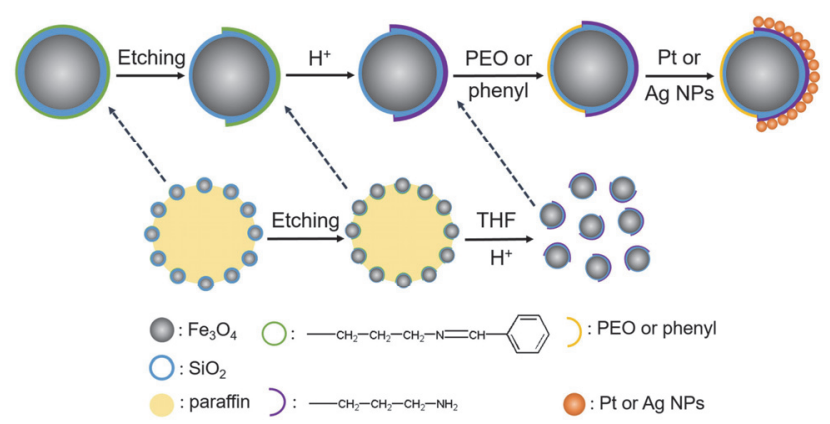

图 $1 \mathrm{Pt}$ 或 $\mathrm{Ag}$ 纳米颗粒负载的顺磁性 $\mathrm{Fe}_{3} \mathrm{O}_{4} @ \mathrm{SiO}_{2}$ Janus 颗粒的制备 过程

Figure 1 Preparation of paramagnetic $\mathrm{Fe}_{3} \mathrm{O}_{4} @ \mathrm{SiO}_{2}-\mathrm{Pt}$ or $\mathrm{Ag}$ Janus particles

\section{2 结果与讨论}

首先, 通过溶剂热法制备顺磁性球形 $\mathrm{Fe}_{3} \mathrm{O}_{4}$ 颗粒, 直径约 $200 \mathrm{~nm}$ (图 2a). X 射线衍射(XRD)数据表明, $\mathrm{Fe}_{3} \mathrm{O}_{4}$ 颗粒的峰位置和强度与标准卡 JCPDS 72-2303(图 $2 \mathrm{c}$, 曲线 a)一致, 证明颗粒的化学组成为 $\mathrm{Fe}_{3} \mathrm{O}_{4} . \mathrm{Fe}_{3} \mathrm{O}_{4}$ 颗粒的饱和磁化强度为 $79.6 \mathrm{emu} / \mathrm{g}$, 从磁滞回线上未检 测到剩磁, 证实 $\mathrm{Fe}_{3} \mathrm{O}_{4}$ 颗粒是顺磁性的(图 2d, 曲线 a).

通过溶胶-凝胶反应在 $\mathrm{Fe}_{3} \mathrm{O}_{4}$ 颗粒表面上包覆 $\mathrm{SiO}_{2}$, 得到核壳结构 $\mathrm{Fe}_{3} \mathrm{O}_{4} @ \mathrm{SiO}_{2}$ 颗粒(图 2b). $\mathrm{SiO}_{2}$ 壳层均匀, 厚度约为 $50 \mathrm{~nm}$. XRD 数据表明 $\mathrm{Fe}_{3} \mathrm{O}_{4} @ \mathrm{SiO}_{2}$ 颗粒中的 $\mathrm{SiO}_{2}$ 为无定形结构, $\mathrm{Fe}_{3} \mathrm{O}_{4}$ 未受影响 (图 $2 \mathrm{c}$, 曲线 b). 磁滞 回线表明 $\mathrm{Fe}_{3} \mathrm{O}_{4} @ \mathrm{SiO}_{2}$ 颗粒的饱和磁化强度为 55.4 $\mathrm{emu} / \mathrm{g}$, 仍为顺磁性(图 2d, 曲线 b).
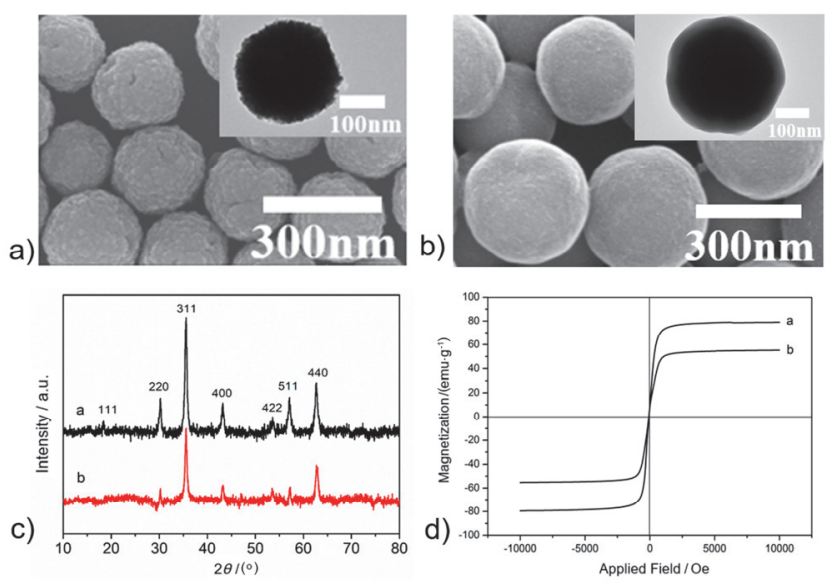

图 2 (a) 顺磁性 $\mathrm{Fe}_{3} \mathrm{O}_{4}$ 颗粒的 SEM 照片和 TEM 照片. (b) $\mathrm{Fe}_{3} \mathrm{O}_{4} @ \mathrm{SiO}_{2}$ 颗粒的 SEM 照片和 TEM 照片. (c) $\mathrm{Fe}_{3} \mathrm{O}_{4}$ 颗粒和 $\mathrm{Fe}_{3} \mathrm{O}_{4} @ \mathrm{SiO}_{2}$ 颗粒的 XRD 图谱. (d) 顺磁性 $\mathrm{Fe}_{3} \mathrm{O}_{4}$ 颗粒(曲线 a) 和 $\mathrm{Fe}_{3} \mathrm{O}_{4} @ \mathrm{SiO}_{2}$ 颗粒(曲线 b) 的磁滞回线

Figure 2 (a) SEM and inset TEM images of the paramagnetic $\mathrm{Fe}_{3} \mathrm{O}_{4}$ particles. (b) SEM and inset TEM images of the $\mathrm{Fe}_{3} \mathrm{O}_{4} @ \mathrm{SiO}_{2}$ particles. (c) X-ray powder diffraction spectra of the $\mathrm{Fe}_{3} \mathrm{O}_{4}$ particles (curve a) and $\mathrm{Fe}_{3} \mathrm{O}_{4} @ \mathrm{SiO}_{2}$ particles (curve b). (d) The magnetic hysteresis loops of the $\mathrm{Fe}_{3} \mathrm{O}_{4}$ particles (curve a) and $\mathrm{Fe}_{3} \mathrm{O}_{4} @ \mathrm{SiO}_{2}$ particles (curve b) 
$\mathrm{Fe}_{3} \mathrm{O}_{4} @ \mathrm{SiO}_{2}$ 颗粒表面为亲水性硅羟基, 作为固体 乳化剂所形成的 Pickering 乳液不稳定. 因此, 使用(3氨基丙基)三甲氧基硅烷(APTES)对其表面进行氨基改 性，再通过苯甲醛与氨基之间的席夫碱反应引入苯甲 醛, 调控颗粒表面的浸润性. 傅里叶变换红外光谱 (FT-IR)光谱中 $567 \mathrm{~cm}^{-1}$ 特征峰为 $\mathrm{Fe}-\mathrm{O}$ 的振动吸收峰, $1080 \mathrm{~cm}^{-1}$ 特征峰为 $\mathrm{Si}-\mathrm{O}-\mathrm{Si}$ 的不对称伸缩振动峰, 分别 归属于 $\mathrm{Fe}_{3} \mathrm{O}_{4}$ 和 $\mathrm{SiO}_{2}$ (图 3, 曲线 $\mathrm{a}, \mathrm{b}$ ). APTES 改性后, 出 现的 $2981 \mathrm{~cm}^{-1}$ 和 $2803 \mathrm{~cm}^{-1}$ 处特征峰归属于 $-\mathrm{CH}_{2}$ 的对 称伸缩振动和反对称伸缩振动, 在 $1573 \mathrm{~cm}^{-1}$ 处的吸收 峰归属于 $-\mathrm{NH}_{2}$ 的面内弯曲振动, 而 $1317 \mathrm{~cm}^{-1}$ 处的吸收 峰归属于 $\mathrm{C}-\mathrm{N}$ 键的伸缩振动, 表明颗粒修饰上氨基(图 3 , 曲线 c). 进一步与苯甲醛反应后在 $1608 \sim 1382 \mathrm{~cm}^{-1}$ 处出现吸收峰, 对应于苯环的骨架振动, 在 $725 \mathrm{~cm}^{-1}$ 处 的吸收峰对应于芳香族 $\mathrm{C}-\mathrm{H}$ 键的伸缩振动, 证明苯甲 醛修饰成功(图 3, 曲线 d).

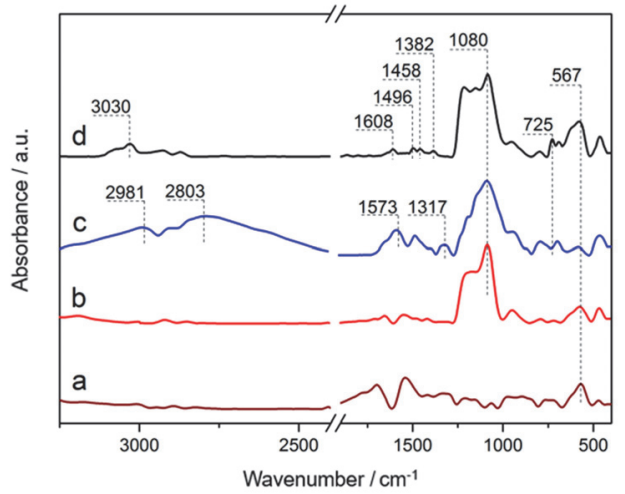

图 3 磁性颗粒的红外光谱图. (a) $\mathrm{Fe}_{3} \mathrm{O}_{4}$ 颗粒; (b) $\mathrm{Fe}_{3} \mathrm{O}_{4} @ \mathrm{SiO}_{2}$ 颗粒; (c) $\mathrm{Fe}_{3} \mathrm{O}_{4} @ \mathrm{SiO}_{2} @ \mathrm{NH}_{2}$ 颗粒; (d) 苯甲醛改性的 $\mathrm{Fe}_{3} \mathrm{O}_{4} @ \mathrm{SiO}_{2}$ 颗粒

Figure 3 FTIR spectra of magnetic particles. (a) $\mathrm{Fe}_{3} \mathrm{O}_{4}$ particles; (b) $\mathrm{Fe}_{3} \mathrm{O}_{4} @ \mathrm{SiO}_{2}$ particles; (c) $\mathrm{Fe}_{3} \mathrm{O}_{4} @ \mathrm{SiO}_{2} @ \mathrm{NH}_{2}$ particles; (d) benzaldehyde modified $\mathrm{Fe}_{3} \mathrm{O}_{4} @ \mathrm{SiO}_{2}$ particles

苯甲醛改性的 $\mathrm{Fe}_{3} \mathrm{O}_{4} @ \mathrm{SiO}_{2}$ 颗粒作为固体乳化剂, 用来稳定水包石蜡乳液. 为了形成 Pickering 乳液, 将颗 粒分散在水中当作水相, 将石蜡融化后当作油相加入到 水中, $\mathrm{Fe}_{3} \mathrm{O}_{4} @ \mathrm{SiO}_{2}$ 颗粒、油相和水相的质量比为 1 : $15: 100$. 在 $70{ }^{\circ} \mathrm{C}$ 高速剪切下形成稳定的 Pickering 乳 液, 乳液可通过磁场进行分离(图 4a). 偏光显微镜图像 显示, 石蜡液滴的直径为 50 100 $\mu \mathrm{m}$ (图 4b). SEM 显示 $\mathrm{Fe}_{3} \mathrm{O}_{4} @ \mathrm{SiO}_{2}$ 颗粒部分嵌入石蜡液滴并在其表面呈单层 结构(图 4c, 4d).

苯甲醛改性前, $\mathrm{Fe}_{3} \mathrm{O}_{4} @ \mathrm{SiO}_{2}$ 颗粒在水中分散良好, 疏水改性后颗粒在水中的分散性差导致团聚(图 5a). $\mathrm{Fe}_{3} \mathrm{O}_{4} @ \mathrm{SiO}_{2}$ 颗粒嵌入石蜡的一侧可以被选择性地保护, 而另一侧暴露在水相, 通过选择性地蚀刻面对水相的一 侧, 从而形成不对称的 Janus 结构. 将石蜡液滴分散在 $\mathrm{NH}_{4} \mathrm{~F}$ 水溶液中, $\mathrm{NH}_{4} \mathrm{~F}$ 蚀刻颗粒 $1 \mathrm{~h}$, 被刻蚀区域表面暴 露硅差基, 用四氢呋喃( THF) 溶解并去除石蜡得到 $\mathrm{Fe}_{3} \mathrm{O}_{4} @ \mathrm{SiO}_{2}$ Janus 颗粒. 扫描电子显微镜( $\mathrm{SEM}$ )和透射 a)
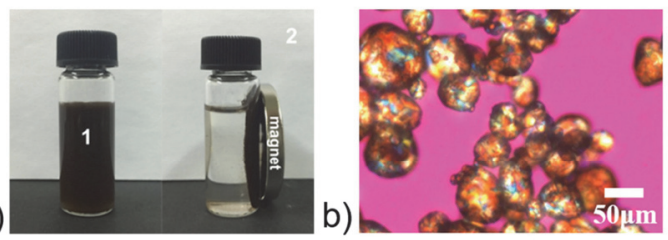

c)
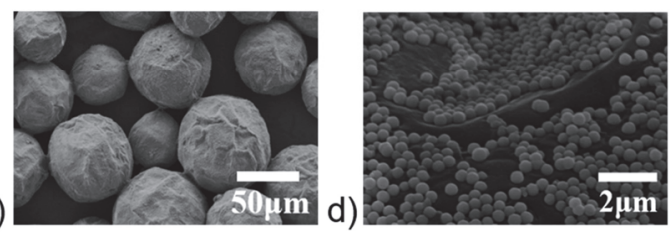

图 4 (a) 苯甲醛改性的 $\mathrm{Fe}_{3} \mathrm{O}_{4} @ \mathrm{SiO}_{2}$ 颗粒乳化的 Pickering 乳液照片. (b) 苯甲醛改性的 $\mathrm{Fe}_{3} \mathrm{O}_{4} @ \mathrm{SiO}_{2}$ 颗粒乳化的 Pickering 乳液的偏光显微镜 图像. (c) 苯甲醛改性的 $\mathrm{Fe}_{3} \mathrm{O}_{4} @ \mathrm{SiO}_{2}$ 颗粒乳化的 Pickering 乳液液滴的 SEM 图像. (d) 苯甲醛改性的 $\mathrm{Fe}_{3} \mathrm{O}_{4} @ \mathrm{SiO}_{2}$ 颗粒乳化的 Pickering 乳液 液滴表面的 SEM 图像

Figure 4 (a) Photograph of Pickering emulsion emulsified with benzaldehyde modified $\mathrm{Fe}_{3} \mathrm{O}_{4} @ \mathrm{SiO}_{2}$ particles. (b) Polarized microscopic image of Pickering emulsion emulsified with benzaldehyde modified $\mathrm{Fe}_{3} \mathrm{O}_{4} @ \mathrm{SiO}_{2}$ particles. (c) $\mathrm{SEM}$ image of Pickering emulsion paraffin droplets emulsified with benzaldehyde modified $\mathrm{Fe}_{3} \mathrm{O}_{4} @ \mathrm{SiO}_{2}$ particles. (d) SEM image of benzaldehyde modified Janus particles on the surface of paraffin droplets a)

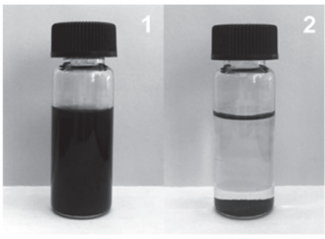

c)

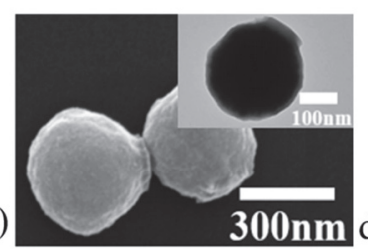

b)

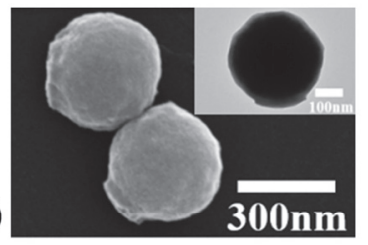

e)

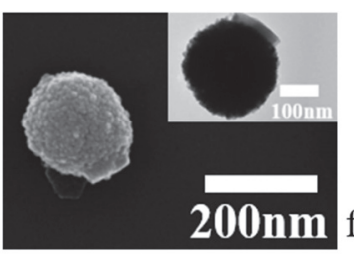

g)

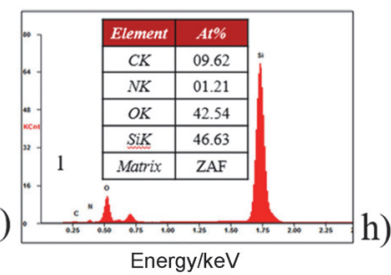

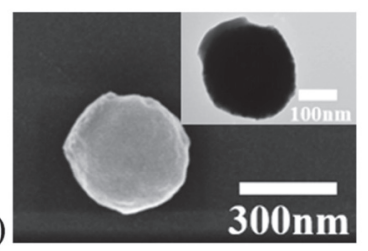
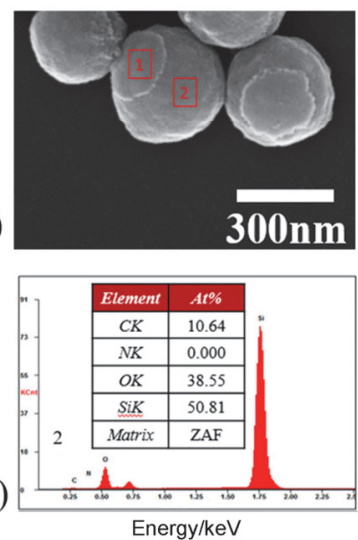

图 5 (a) 苯甲醛改性前后 $\mathrm{Fe}_{3} \mathrm{O}_{4} @ \mathrm{SiO}_{2}$ 颗粒在水中的分散情况: (1) 改性前, (2) 改性后. 不同刻蚀时间下 $\mathrm{Fe}_{3} \mathrm{O}_{4} @ \mathrm{SiO}_{2}$ Janus 颗粒的 SEM 和 TEM 图像, 其中刻蚀时间分别为(b) 1 h, (c) 2 h, (d) 3 h, (e) 4 h. (f h) 刻蚀后的 $\mathrm{Fe}_{3} \mathrm{O}_{4} @ \mathrm{SiO}_{2}$ 颗粒表面不同区域的 $\mathrm{EDX}$ 元素分析图谱.

Figure 5 (a) Photograph of the dispersion of $\mathrm{Fe}_{3} \mathrm{O}_{4} @ \mathrm{SiO}_{2}$ particles in water (1) before and (2) after benzaldehyde modification. SEM and inset TEM images of $\mathrm{Fe}_{3} \mathrm{O}_{4} @ \mathrm{SiO}_{2}$ Janus particles at different etching times: (b) $1 \mathrm{~h}$, (c) $2 \mathrm{~h}$, (d) $3 \mathrm{~h}$, (e) $4 \mathrm{~h}$. (f $\sim \mathrm{h})$ EDX elemental analysis of different areas of the etched $\mathrm{Fe}_{3} \mathrm{O}_{4} @ \mathrm{SiO}_{2}$ Janus particle surface 
电子显微镜(TEM)显示刻蚀面较浅, $\mathrm{SiO}_{2}$ 层被刻蚀 30 $40 \mathrm{~nm}$, 颗粒显出不对称形状(图 5b). 随着蚀刻时间增 加, $\mathrm{SiO}_{2}$ 壳层被进一步刻蚀(图 5c,5d). 当蚀刻时间延长 至 $4 \mathrm{~h}$ 时, 在水相裸露的 $\mathrm{SiO}_{2}$ 壳层被完全蚀刻, 暴露出 $\mathrm{Fe}_{3} \mathrm{O}_{4}$ 表面(图 5e). 当蚀刻时间为 $2 \mathrm{~h}$ 时, 能谱分析 $(\mathrm{EDX})$ 结果表明在未被刻蚀一侧检测到 $\mathrm{N}$ 元素, 证明氨 基未受影响; 而被刻蚀一侧没有 $\mathrm{N}$ 元素, 证明氨基被完 全刻蚀去除 (图 5f 5h). 这证明了所制备的非对称 $\mathrm{Fe}_{3} \mathrm{O}_{4} @ \mathrm{SiO}_{2}$ 颗粒具有 Janus 结构, 并且 Janus 颗粒的非 对称结构能够通过刻蚀时间进行调控.

利用 2-[甲氧基(聚乙烯氧基) 6-9 丙基]-三甲氧基硅烷 对 $\mathrm{Fe}_{3} \mathrm{O}_{4} @ \mathrm{SiO}_{2}$ Janus 颗粒被刻蚀一侧进行改性, 修饰 PEO 链段. FT-IR 光谱显示在 $1107 \mathrm{~cm}^{-1}$ 处有 C-O-C 的拉 伸振动吸收峰, 表明实现了 PEO 改性(图 6). 经 PEO 改 性后, 被刻蚀的 $\mathrm{Fe}_{3} \mathrm{O}_{4} @ \mathrm{SiO}_{2}$ Janus 颗粒的表面仍然相对 光滑, 没有明显变化(图 7a). 当 $\mathrm{Fe}_{3} \mathrm{O}_{4} @ \mathrm{SiO}_{2}$ Janus 颗粒

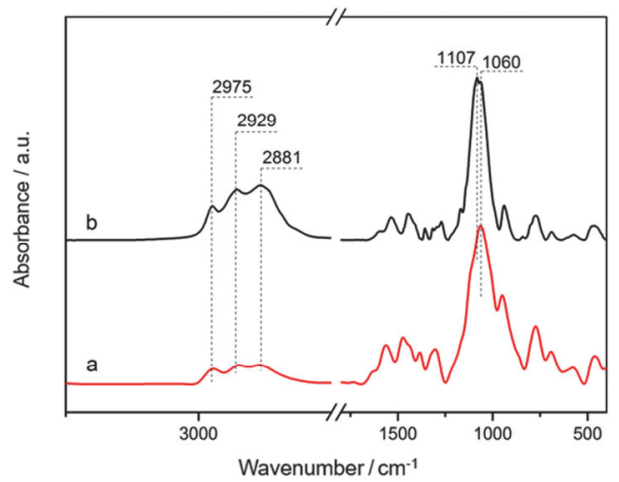

图 6 颗粒的 FT-IR 光谱: (a) 刻蚀的 $\mathrm{Fe}_{3} \mathrm{O}_{4} @ \mathrm{SiO}_{2}$ Janus 颗粒; (b) PEO 改性后的 $\mathrm{Fe}_{3} \mathrm{O}_{4} @ \mathrm{SiO}_{2}$ Janus 颗粒

Figure 6 FT-IR spectra of the particles: (a) etched $\mathrm{Fe}_{3} \mathrm{O}_{4} @ \mathrm{SiO}_{2}$ Janus particles; (b) PEO segment modified $\mathrm{Fe}_{3} \mathrm{O}_{4} @ \mathrm{SiO}_{2}$ Janus particles

a)
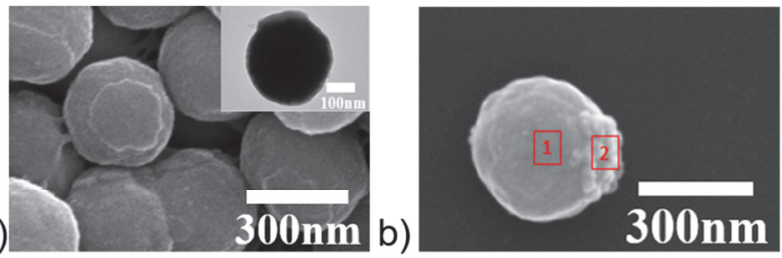

C)

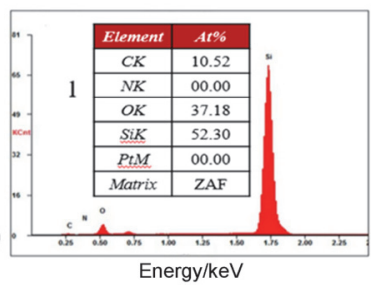

d)

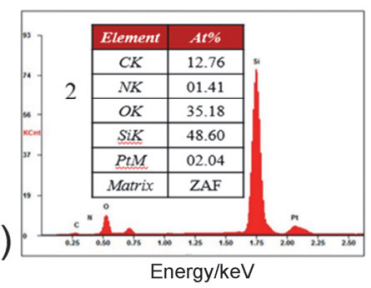

图 7 (a) PEO 链段改性的 $\mathrm{Fe}_{3} \mathrm{O}_{4} @ \mathrm{SiO}_{2} \mathrm{Janus}$ 颗粒的 SEM 和 TEM 照 片. (b) $\mathrm{Fe}_{3} \mathrm{O}_{4} @ \mathrm{SiO}_{2}$-Pt Janus 颗粒的 SEM 照片. (c, d) $\mathrm{Fe}_{3} \mathrm{O}_{4} @ \mathrm{SiO}_{2}-\mathrm{Pt}$ Janus 颗粒表面不同区域的 EDX 元素分析图谱

Figure 7 (a) SEM and inset TEM images of $\mathrm{Fe}_{3} \mathrm{O}_{4} @ \mathrm{SiO}_{2}$ Janus particles modified by PEO segment. (b) SEM image of $\mathrm{Fe}_{3} \mathrm{O}_{4} @ \mathrm{SiO}_{2}$-Pt Janus particles. (c, d) EDX elemental analysis of different areas of $\mathrm{Fe}_{3} \mathrm{O}_{4} @ \mathrm{SiO}_{2}-\mathrm{Pt}$ Janus particles
分散在 $\mathrm{H}_{2} \mathrm{PtCl}_{6} \cdot 6 \mathrm{H}_{2} \mathrm{O}$ 水溶液中，未刻蚀一侧上的氨基与 $\mathrm{Pt}^{4+}$ 发生配位作用而吸附 $\mathrm{Pt}^{4+}$, 而被刻蚀的一侧被 PEO 链段保护没有吸附 $\mathrm{Pt}^{4+}$. $\mathrm{NaBH}_{4}$ 作为还原剂将 $\mathrm{Pt}^{4+}$ 还原 为 $\mathrm{Pt}$ 纳米颗粒. $\mathrm{SEM}$ 显示 $\mathrm{Pt}$ 纳米颗粒仅复合在氨基一 侧, 而另一侧则没有 Pt 纳米颗粒(图 7b). EDX 结果进一 步证明 $\mathrm{Pt}$ 纳米颗粒仅复合在氨基一侧(图 7c, 7d).

在 $\mathrm{Pt}$ 纳米颗粒的催化下, $\mathrm{H}_{2} \mathrm{O}_{2}$ 能够分解生成 $\mathrm{H}_{2} \mathrm{O}$ 和 $\mathrm{O}_{2}$ 推动颗粒做自驱动运动. Ozin 等 ${ }^{[44]}$ 通过表面溅射 和热退火制备了由 $\mathrm{Pt}$ 和 $\mathrm{SiO}_{2}$ 组成的雪人状的 Janus 颗 粒, 研究发现 $\mathrm{SiO}_{2} @ \mathrm{Pt}$ Janus 颗粒在 $\mathrm{H}_{2} \mathrm{O}_{2}$ 水溶液中的运 动可分为两种: 类线性运动和类圆周旋转.

由于不对称的化学反应, $\mathrm{Fe}_{3} \mathrm{O}_{4} @ \mathrm{SiO}_{2}-\mathrm{Pt}$ Janus 颗粒 具有自驱动作用. 将 $\mathrm{Fe}_{3} \mathrm{O}_{4} @ \mathrm{SiO}_{2}-\mathrm{Pt}$ Janus 颗粒分散在 不同浓度的 $\mathrm{H}_{2} \mathrm{O}_{2}$ 水溶液中, 通过光学显微镜观察颗粒 的运动. 当颗粒分散在水中时, 运动轨迹表明颗粒仅具 有随机的布朗运动且幅度较小 (图 $8 \mathrm{a}, 8 \mathrm{~b}$ ). 当分散在质 量分数 $1.0 \%$ 的 $\mathrm{H}_{2} \mathrm{O}_{2}$ 水溶液时, 颗粒的运动变得强烈, 运动轨迹范围变大(图 $8 \mathrm{c}, 8 \mathrm{~d}$ ), 这表明剧烈的运动是由 自驱动而不是布朗运动引起的. 当 $\mathrm{H}_{2} \mathrm{O}_{2}$ 水溶液的浓度 增加到质量分数 $5.0 \%$ 时, 颗粒移动的速度更快, 运动的 轨迹范围变得更大 (图 $8 \mathrm{e}, 8 \mathrm{f}$ ). 根据统计计算, $\mathrm{Fe}_{3} \mathrm{O}_{4} @ \mathrm{SiO}_{2}-\mathrm{Pt}$ Janus 颗粒在水中和质量分数 1.0\%和 $5.0 \% \mathrm{H}_{2} \mathrm{O}_{2}$ 水溶液中的平均速度分别为 $0.4,1.3$ 和 2.9 $\mu \mathrm{m} / \mathrm{s}$. a)

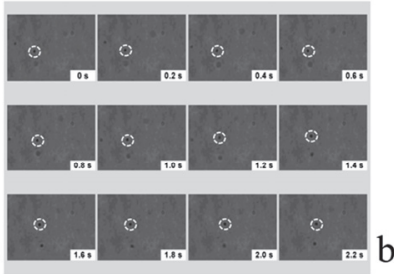

c)

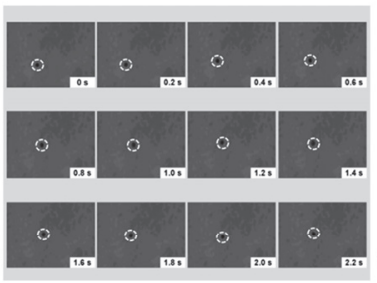

d)

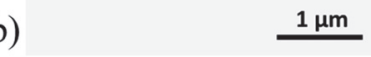

e)

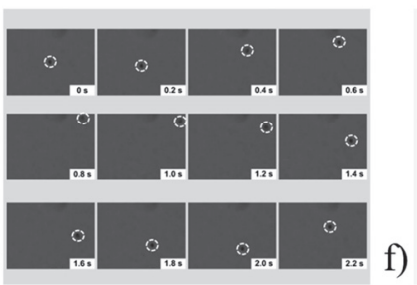

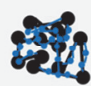

$1 \mu \mathrm{m}$
图 $8 \mathrm{Fe}_{3} \mathrm{O}_{4} @ \mathrm{SiO}_{2}-\mathrm{Pt}$ Janus 颗粒在不同溶液中的运动视频截图和运动 轨迹图(其中每张图时间间隔为 $200 \mathrm{~ms}$ ). (a, b) 水; (c, d) 质量分数 $1.0 \%$ 的过氧化氢溶液; (e, f) 质量分数 $5.0 \%$ 的过氧化氢溶液

Figure 8 Motion video shots and motion traces of the $\mathrm{Fe}_{3} \mathrm{O}_{4} @ \mathrm{SiO}_{2}-\mathrm{Pt}$ Janus particles (with intervals of $200 \mathrm{~ms}$ in each image). (a, b) water; (c, d) mass fraction $1.0 \% \mathrm{H}_{2} \mathrm{O}_{2}$ aqueous solution; (e, f) mass fraction $5.0 \%$ $\mathrm{H}_{2} \mathrm{O}_{2}$ aqueous solution 
类似地, $\mathrm{Fe}_{3} \mathrm{O}_{4} @ \mathrm{SiO}_{2}-\mathrm{Ag}$ Janus 颗粒通过选择修饰和 复合 $\mathrm{Ag}$ 纳米粒子制备. 首先, 用苯基三乙氧基硅烷对 被刻蚀一侧进行疏水改性, 一方面是防止由于静电作用 引起 $\mathrm{Ag}^{+}$吸附, 另一方面对 Janus 颗粒进行疏水改性赋 予其两亲性. 经苯基改性后, 被刻蚀的一侧表面没有明 显变化(图 9a). 当 $\mathrm{Fe}_{3} \mathrm{O}_{4} @ \mathrm{SiO}_{2}$ Janus 颗粒分散在 $\mathrm{AgNO}_{3}$ 水溶液中, 氨基一侧与 $\mathrm{Ag}^{+}$配位, 被刻蚀的一侧被苯基 保护则不进行配位. 进一步用 $\mathrm{NaBH}_{4}$ 将 $\mathrm{Ag}^{+}$还原成 $\mathrm{Ag}$ 纳米颗粒, 得到 $\mathrm{Fe}_{3} \mathrm{O}_{4} @ \mathrm{SiO}_{2}-\mathrm{Ag} \mathrm{Janus}$ 颗粒, $\mathrm{Ag}$ 纳米颗 粒仅在氨基一侧复合(图 9b). 两亲性的 $\mathrm{Fe}_{3} \mathrm{O}_{4} @ \mathrm{SiO}_{2}-\mathrm{Ag}$ Janus 颗粒作为固体乳化剂可以稳定油包水型乳液(图 9c). 为探究 Janus 颗粒在乳液界面的排布, 水相和 Janus 颗粒分别被异硫氰(酸荧光素(FITC)染料和羧基罗丹明 染色, 在激光扫描共聚焦显微镜(CLSM) 图像中分别显 示为蓝色和绿色, 可以清楚地看到 $\mathrm{Fe}_{3} \mathrm{O}_{4} @ \mathrm{SiO}_{2}-\mathrm{Ag} \mathrm{Ja}-$ nus 颗粒集中在油水界面, 乳液的平均液滴直径约为 100 200 $\mu \mathrm{m}$ (图 9d). a)

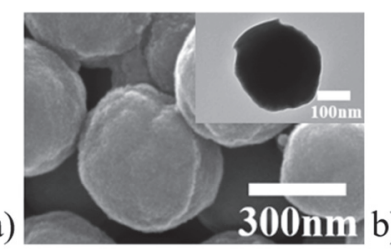

c)

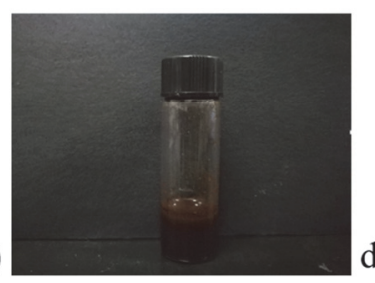

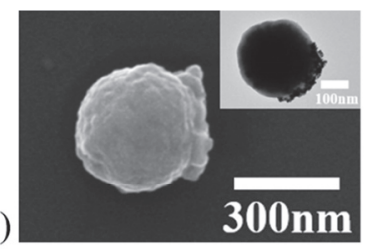

d)

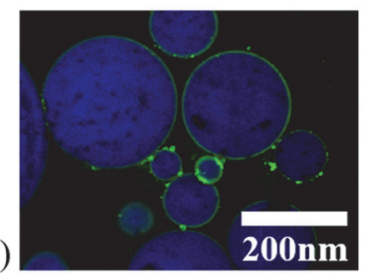

图 9 (a) 苯基改性的 $\mathrm{Fe}_{3} \mathrm{O}_{4} @ \mathrm{SiO}_{2}$ Janus 颗粒的 SEM 和 TEM 照片. (b) $\mathrm{Fe}_{3} \mathrm{O}_{4} @ \mathrm{SiO}_{2}-\mathrm{Ag}$ Janus 颗粒的 SEM 和 TEM 照片. (c) $\mathrm{Fe}_{3} \mathrm{O}_{4} @ \mathrm{SiO}_{2}-\mathrm{Ag}$ Janus 颗粒稳定的 4-硝基苯甲醚的甲苯溶液和 $\mathrm{NaBH}_{4}$ 水溶液的乳液照 片. (d) 乳液的 CLSM 图像

Figure 9 (a) SEM and inset TEM images of phenyl modified $\mathrm{Fe}_{3} \mathrm{O}_{4} @ \mathrm{SiO}_{2}$ Janus particles. (b) SEM and inset TEM images of $\mathrm{Fe}_{3} \mathrm{O}_{4} @ \mathrm{SiO}_{2}-\mathrm{Ag}$ Janus particles. (c) The photograph of the water-in-toluene emulsion stabilized with $\mathrm{Fe}_{3} \mathrm{O}_{4} @ \mathrm{SiO}_{2}-\mathrm{Ag}$ Janus particles. (d) The CLSM image of emulsion stabilized with $\mathrm{Fe}_{3} \mathrm{O}_{4} @ \mathrm{SiO}_{2}-\mathrm{Ag}$ Janus particles

$\mathrm{Fe}_{3} \mathrm{O}_{4} @ \mathrm{SiO}_{2}-\mathrm{Ag}$ Janus 颗粒既可用作固体乳化剂, 又可用作催化剂定位到乳液油水界面. 以 4-硝基苯甲醚 的甲苯溶液作为油相, $\mathrm{NaBH}_{4}$ 水溶液作为水相, 将 $\mathrm{Fe}_{3} \mathrm{O}_{4} @ \mathrm{SiO}_{2}-\mathrm{Ag}$ Janus 颗粒分散在上述溶液中高速剪切 $2 \mathrm{~min}$ 得到乳液. $\mathrm{Ag}$ 纳米粒子可以催化硝基化合物的还 原 ${ }^{[45]}, \mathrm{Fe}_{3} \mathrm{O}_{4} @ \mathrm{SiO}_{2}-\mathrm{Ag}$ Janus 颗粒在乳液界面的反应过 程如下(图 10). 4-硝基苯甲醚和 4-氨基苯甲醚的特征峰 分别在 $304 \mathrm{~nm}$ 和 $309 \mathrm{~nm}$ 处(图 11a, 11b), 随着反应的进 行，4-硝基苯甲醚在 $304 \mathrm{~nm}$ 处的吸收峰逐渐减弱, 而 $309 \mathrm{~nm}$ 处吸收峰随之增强, 证明 4-硝基苯甲醚被还原 成 4-氨基苯甲醚. 在没有 Janus 颗粒的情况下将 $\mathrm{NaBH}_{4}$ 加到 4-硝基苯甲醚的甲苯溶液中，4-硝基苯甲醚特征峰
的位置和强度几乎不变，证明 $\mathrm{NaBH}_{4}$ 不溶于甲苯没有 发挥还原剂的作用，证明了 $\mathrm{Fe}_{3} \mathrm{O}_{4} @ \mathrm{SiO}_{2}-\mathrm{Ag}$ Janus 颗粒 的催化作用. 根据 4-硝基苯甲醚和 4-氨基苯甲醚的标准 曲线和反应进行的样品的紫外强度，可以计算出 4-硝基 苯甲醚的转化率(图 11c, 11d).

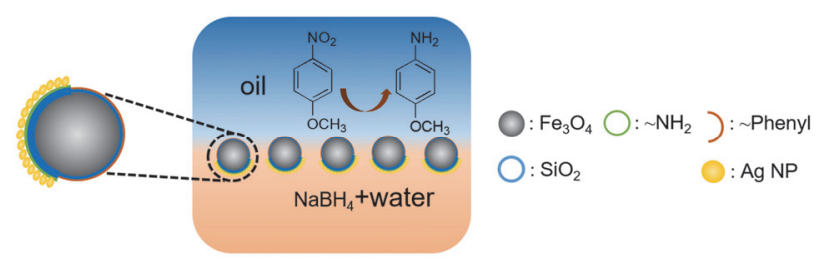

图 $10 \mathrm{Fe}_{3} \mathrm{O}_{4} @ \mathrm{SiO}_{2}-\mathrm{Ag}$ Janus particles 在乳液界面的催化还原过程 Figure 10 The emulsion interfacial catalysis of $\mathrm{Fe}_{3} \mathrm{O}_{4} @ \mathrm{SiO}_{2}-\mathrm{Ag}$ Janus particles
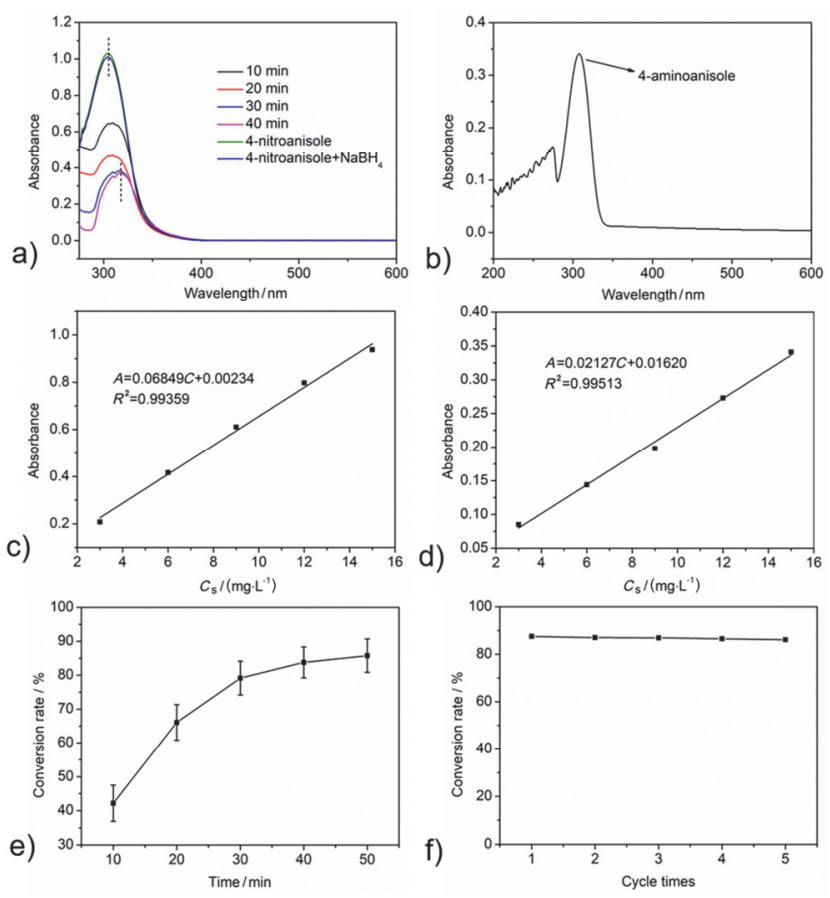

图 $11 \mathrm{Fe}_{3} \mathrm{O}_{4} @ \mathrm{SiO}_{2}-\mathrm{Ag}$ Janus 颗粒作为固体乳化剂对 4-硝基苯甲醚的 催化. (a) 4-硝基苯甲醚在不同时间内的 UV-vis: $10 \mathrm{~min}$ (黑色曲线), 20 $\min$ (红色曲线), $30 \mathrm{~min}$ (浅蓝色曲线), $40 \mathrm{~min}$ (粉色曲线), $9.8 \times 10^{-5}$ $\mathrm{mol} / \mathrm{L}$ 4-硝基苯甲醚(绿色曲线), 不加 $\mathrm{NaBH}_{4}$ 的体系(深蓝色曲线). (b) 4-氨基苯甲醚的 UV-vis. (c, d) 4-硝基苯甲醚和 4-氨基苯甲醚的标准曲 线. (e) 4-硝基苯甲梄在乳液体系中的反应动力学研究. (f) $\mathrm{Fe}_{3} \mathrm{O}_{4} @ \mathrm{SiO}_{2}-\mathrm{Ag}$ Janus 颗粒的循环使用

Figure 11 (a) UV-vis spectra of 4-nitroanisole concentration in the water-in-oil emulsion after conversion by $\mathrm{Fe}_{3} \mathrm{O}_{4} @ \mathrm{SiO}_{2}-\mathrm{Ag}$ Janus particles with different time: $10 \mathrm{~min}$ (curve black), $20 \mathrm{~min}$ (curve red), $30 \mathrm{~min}$ (curve light blue), $40 \mathrm{~min}$ (curve pink), $9.8 \times 10^{-5} \mathrm{~mol} / \mathrm{L}$ 4-nitroanisole (curve green), the system without $\mathrm{NaBH}_{4}$ (curve navy blue). (b) UV-Vis spectrum of 4-aminoanisole. (c, d) The standard curve of 4-nitroanisole and 4-aminoanisole. (e) Conversion kinetics of 4-nitroanisole in emulsion system. (f) Catalyst recycling of $\mathrm{Fe}_{3} \mathrm{O}_{4} @ \mathrm{SiO}_{2}-\mathrm{Ag}$ Janus particles in the emulsion system

$$
\begin{array}{ll}
A_{1}=0.06849 C_{1}+0.00234 & \text { (4-硝基苯甲醚的标准曲线) } \\
A_{2}=0.02127 C_{2}+0.01620 & \text { (4-氨基苯甲醚的标准曲线) }
\end{array}
$$




$$
\begin{gathered}
A_{\text {总 }}=A_{1}+A_{2}=0.06849 C_{1}+0.02127 C_{2}+0.01854 \\
C_{2}=\left(15-C_{1}\right) / 153 * 120=11.7647-0.7843 C_{1} \\
\quad M_{\text {确基 }}=153, M_{\text {氮基 }}=120, \text { 将式 }(2) \text { 代入式 }(1) \\
A \text { 总 }=A_{1}+A_{2}=0.05181 C_{1}+0.2687, \text { 根据不同时间的 }
\end{gathered}
$$
强度计算可得 $C_{1}$ 转化率 $(\%)=\left(C_{\mathrm{o}}-C_{1}\right) / C_{\mathrm{o}} \times 100 \%$

反应 $40 \mathrm{~min}$, 转化率为 $87.5 \%$ (图 11e). 进一步延长 反应时间, 转化率没有明显提高. 通过外加磁场能够从 乳液中回收 $\mathrm{Fe}_{3} \mathrm{O}_{4} @ \mathrm{SiO}_{2}-\mathrm{Ag}$ Janus 颗粒, 用乙醇和水洗 涤后可重新获得颗粒, 经过 5 次催化循环后, 转化率从 $87.5 \%$ 降低到 $86.1 \%$, 仍然表现出较高的催化效率(图 11f).

\section{3 结论}

通过 Pickering 乳液和选择性蚀刻方法成功制备了 $\mathrm{Fe}_{3} \mathrm{O}_{4} @ \mathrm{SiO}_{2}$ Janus 颗粒, 进一步通过选择性生长 Pt 和 $\mathrm{Ag}$ 纳米颗粒分别得到 $\mathrm{Fe}_{3} \mathrm{O}_{4} @ \mathrm{SiO}_{2}-\mathrm{Pt}$ 和 $\mathrm{Fe}_{3} \mathrm{O}_{4} @ \mathrm{SiO}_{2}-\mathrm{Ag}$ Janus 颗粒. 由于不对称的结构和性质, $\mathrm{Fe}_{3} \mathrm{O}_{4} @ \mathrm{SiO}_{2}-\mathrm{Pt}$ Janus 颗粒在催化 $\mathrm{H}_{2} \mathrm{O}_{2}$ 分解成水和氧气的体系中可实现 颗粒的自驱动. 两亲性的 $\mathrm{Fe}_{3} \mathrm{O}_{4} @ \mathrm{SiO}_{2}-\mathrm{Ag}$ Janus 颗粒在 稳定乳液的同时, 将催化功能引入界面反应实现 4-硝基 苯甲醚的高效催化.

\section{4 实验部分}

\section{$4.1 \mathrm{Fe}_{3} \mathrm{O}_{4} @ \mathrm{SiO}_{2}$ 颗粒的制备}

通过溶剂热法制备顺磁性 $\mathrm{Fe}_{3} \mathrm{O}_{4}$ 颗粒 ${ }^{[46]}$. 将 $2.7 \mathrm{~g}$ $\mathrm{FeCl}_{3} \cdot 6 \mathrm{H}_{2} \mathrm{O}$ 分散到 $100 \mathrm{~mL}$ 乙二醇( $\left.\mathrm{EG}\right)$ 中, 溶解后将 $7.2 \mathrm{~g} \mathrm{NaAc}$ 加到上述溶液, 超声 $30 \mathrm{~min}$ 后获得黄色的均 匀溶液. 将溶液转移到反应金中 $200{ }^{\circ} \mathrm{C}$ 下反应 $12 \mathrm{~h}$, 用 乙醇和水洗涤 3 次后获得顺磁性的 $\mathrm{Fe}_{3} \mathrm{O}_{4}$ 颗粒.

在超声处理下, 将 $0.1 \mathrm{~g}$ 的 $\mathrm{Fe}_{3} \mathrm{O}_{4}$ 颗粒分散到 $50 \mathrm{~mL}$ 的 $0.1 \mathrm{~mol} / \mathrm{L} \mathrm{HCl}$ 溶液中, $10 \mathrm{~min}$ 后用磁铁分离、水洗 3 次得到 $\mathrm{Fe}_{3} \mathrm{O}_{4}$ 颗粒. 然后, 将颗粒分散到 $50 \mathrm{~mL}$ 质量分 数 $20 \%$ 柠檬酸钠溶液中, 超声处理 $30 \mathrm{~min}$ 后用乙醇和水 洗涤 3 次得到颗粒. 将处理后的 $\mathrm{Fe}_{3} \mathrm{O}_{4}$ 颗粒、 $5 \mathrm{~mL}$ 水 和 $1.5 \mathrm{~mL} \mathrm{NH}_{3} \cdot \mathrm{H}_{2} \mathrm{O}$ 加入到 $60 \mathrm{~mL}$ 乙醇中, 搅拌 $1 \mathrm{~h}$ 后将 一定量的正硅酸乙酯(TEOS)加入到上述溶液中, 反应 8 $\mathrm{h}$ 后用乙醇和水分别洗涤 3 次得到 $\mathrm{Fe}_{3} \mathrm{O}_{4} @ \mathrm{SiO}_{2}$ 颗粒.

将 $0.1 \mathrm{~g} \mathrm{Fe}_{3} \mathrm{O}_{4} @ \mathrm{SiO}_{2}$ 颗粒和 $100 \mu \mathrm{L}$ 3-氨丙基三乙氧 基硅烷(APTES)加入到 $100 \mathrm{~mL}$ 甲苯中, 反应在 $80{ }^{\circ} \mathrm{C}$ 下 回流 $8 \mathrm{~h}$. 冷却至室温后, 用甲苯洗涤 3 次得到 $\mathrm{Fe}_{3} \mathrm{O}_{4} @ \mathrm{SiO}_{2} @ \mathrm{NH}_{2}$ 颗粒. 将该颗粒、50 mg 苯甲醛和 5 $\mu \mathrm{L}$ 冰醋酸加入到 $100 \mathrm{~mL}$ 乙醇中, 该溶液在室温下反应 $12 \mathrm{~h}$, 经乙醇和水分别洗涤 3 次后得到苯甲醛改性的 $\mathrm{Fe}_{3} \mathrm{O}_{4} @ \mathrm{SiO}_{2}$ 颗粒.

\section{2 $\mathrm{Fe}_{3} \mathrm{O}_{4} @ \mathrm{SiO}_{2}$ Janus 颗粒的制备}

在 $70{ }^{\circ} \mathrm{C}$ 下, 将 $0.2 \mathrm{~g}$ 苯甲醛改性的 $\mathrm{Fe}_{3} \mathrm{O}_{4} @ \mathrm{SiO}_{2}$ 颗
粒、 $3 \mathrm{~g}$ 石蜡加入到 $20 \mathrm{~mL}$ 水中, 在 $12000 \mathrm{r} / \mathrm{min}$ 的速度 下搅拌 $5 \mathrm{~min}$. 待乳液冷却至室温, 石蜡乳液凝固. 将乳 液液滴加到 $2 \mathrm{~mL}$ 的质量分数 $5 \%$ 的 $\mathrm{NH}_{4} \mathrm{~F}$ 水溶液中蚀刻 $2 \mathrm{~h}$, 然后用 $\mathrm{THF}$ 洗涤 3 次去除石蜡得到 $\mathrm{Fe}_{3} \mathrm{O}_{4} @ \mathrm{SiO}_{2}$ Janus 颗粒. 将刻蚀得到的 Janus 颗粒和 $200 \mu \mathrm{L} 2$ 2-[甲氧 基(聚乙氧基) 6 -9 丙基]三甲氧基硅烷或苯基三乙氧基硅 烷加入到 $100 \mathrm{~mL}$ THF 中, 反应在 $60{ }^{\circ} \mathrm{C}$ 下搅拌 $12 \mathrm{~h}$, 经 $\mathrm{THF}$ 洗涤 3 次后得到 $\mathrm{PEO}$ 或苯基修饰的 $\mathrm{Fe}_{3} \mathrm{O}_{4} @ \mathrm{SiO}_{2}$ Janus 颗粒.

\section{$4.3 \mathrm{Fe}_{3} \mathrm{O}_{4} @ \mathrm{SiO}_{2}-\mathrm{Pt}$ Janus 颗粒制备}

将 $0.01 \mathrm{~g}$ PEO 修饰的 Janus 颗粒分散在 $10 \mathrm{~mL}$ 的质 量分数 $20 \%$ 的 $\mathrm{H}_{2} \mathrm{PtCl}_{6} \cdot 6 \mathrm{H}_{2} \mathrm{O}$ 水溶液中搅拌 $2 \mathrm{~h}$, 加入 2 $\mathrm{mg} \mathrm{NaBH}_{4}$ 继续搅拌 $2 \mathrm{~h}$ 得到 $\mathrm{Fe}_{3} \mathrm{O}_{4} @ \mathrm{SiO}_{2}-\mathrm{Pt}$ Janus 颗粒.

\section{$4.4 \mathrm{Fe}_{3} \mathrm{O}_{4} @ \mathrm{SiO}_{2}-\mathrm{Ag}$ Janus 颗粒制备}

将 $0.01 \mathrm{~g}$ 上述苯基修饰的 Janus 颗粒分散在 $10 \mathrm{~mL}$ 质量分数 $5 \%$ 的 $\mathrm{AgNO}_{3}$ 水溶液中搅拌 $2 \mathrm{~h}$, 加入 $2 \mathrm{mg}$ $\mathrm{NaBH}_{4}$ 在冰水浴中搅拌 $2 \mathrm{~h}$, 得到 $\mathrm{Fe}_{3} \mathrm{O}_{4} @ \mathrm{SiO}_{2}-\mathrm{Ag}$ Janus 颗粒.

\section{5 催化实验}

将 10 mg $\mathrm{Fe}_{3} \mathrm{O}_{4} @ \mathrm{SiO}_{2}-\mathrm{Ag}$ Janus 颗粒加到 $2 \mathrm{~mL}$ 溶有 $15 \mathrm{ppm}$ 4-硝基苯甲醚的甲苯溶液中，再将 $0.5 \mathrm{~mL}$ 的 $\mathrm{NaBH}_{4}$ 水溶液加到上述混合物中，高速剪切后得到乳 液，在室温下反应 $40 \mathrm{~min}$, 每隔 $10 \mathrm{~min}$ 取出甲苯溶液测 其紫外强度.

\section{References}

[1] De Gennes, P. G. Rev. Mod. Phys. 1992, 64, 645

[2] Jiang, S.; Granick, S. Janus Particles Synthesis, Self-assembly and Applications, RSC, London, England, 2012.

[3] Shi, S. Y.; Zhang, L. L.; Zhang, G. L.; Song, X. M.; Sun, D. Y.; Liang, F. X.; Yang, Z. Z. Macromolecules 2020, 53, 2228.

[4] Xiang, D.; Jiang, B. Y.; Liang, F. X.; Yan, L. T.; Yang, Z. Z. Macromolecules 2020, 53, 1063.

[5] Zhang, H.; Wang, Q.; Jiang, B. Y.; Liang, F. X.; Yang, Z. Z. ACS Appl. Mater. Interfaces 2016, 8, 33250.

[6] Zhao, R. T.; Yu, X. T.; Sun, D. Y.; Huang, L. Y.; Liang, F. X.; Liu, Z. P. ACS Appl. Nano. Mater. 2019, 2, 2127.

[7] Zhao, R. T.; Han, T. H.; Sun, D. Y.; Huang, L. Y.; Liang, F. X.; Liu, Z. P. Langmuir 2019, 35, 11435.

[8] Nisisako, T.; Torii, T.; Takahashi, T.; Takizawa, Y. Adv. Mater. 2006 , $18,1152$.

[9] Hays, D. A. J. Electrost. 2001, 51-52, 57.

[10] Wu, B.; Liu, Z. Q.; Liu, X. S.; Liu, G. Q.; Tang, P.; Yuan, W.; Fu, G. L. Nanotechnology 2020, 31, 225301.

[11] Dolbashian1, C.; Chavez1, B. L.; Bauer, M.; Budi, M.; Andrew, J. S.; Crawford, T. M. J. Phys. D: Appl. Phys. 2020, 53, 195002.

[12] Li, L. L.; Bacaksiz, C.; Nakhaee, M.; Pentcheva, R.; Peeters, F. M.; Yagmurcukardes, M. Phys. Rev. B 2020, 101, 134102.

[13] Paulus, M.; Degen, P.; Brenner, T.; Tiemeyer, S.; Struth, B.; Tolan, M.; Rehage, H. Langmuir 2010, 26, 15945.

[14] Xu, Q. A.; Kang, X. W.; Bogomolni, R. A.; Chen, S. W. Langmuir 2010, 26, 14923.

[15] Ozin, G. A.; Manners, I.; Fournier-Bidoz, S.; Arsenault, A. Adv. Mater. 2005, 17, 3011 .

[16] Wang, J. ACS Nano 2009, 3, 4.

[17] Cui, L. Y.; Fan, S. S.; Yu, C. L. Acta Chim. Sinica 2017, 75, 967 (in Chinese). (崔丽影，范莎莎，于存龙，化学学报, 2017, 75, 967.)

[18] Zhang, B. B.; Ma, C.; Wang, X. G. Acta Chim. Sinica 2015, 73, 441 (in Chinese). (张贝贝, 马驰, 王小刚, 化学学报, 2015, 73, 441.) 
[19] Chen, C. Y.; Yi, J. Q.; Dong, H. Y. Chin. J. Chem. 2015, 33, 527.

[20] Liang, F. X.; Yang, Z. Z. Acta Polym. Sin. 2017, (6), 883 (in Chinese). (梁福金金, 杨振忠, 高分子学报, 2017, (6), 883.)

[21] Tang, L.; Liang, F. X.; Wang, Q. Chin. J. Polvm. Sci. 2017, 35, 799 (in Chinese). (唐琳, 梁福金金, 主倩, 高分子科学, 2017, 35, 799.)

[22] Meng, H. Y.; Wan, J. P.; Jing, J. Y. Chin. Chem. Lett. 2020, 31, 253 (in Chinese). (孟宏宇，万基平，井静云，中国化学快报，2020，31， 253.)

[23] Jing, J. Y.; Yao, X. H.; Yang, Z. Z. Acta Polym. Sin. 2018, 8, 1066 (in Chinese). (井静云, 姚晓辉, 杨振忠, 高分子学报, 2018, 8, 1066.)

[24] Liang, F. X.; Liu, B.; Yang, Z. Z. Polym. Bull. 2016, (9), 45 (in Chinese). (梁福金金，刘冰，杨振忠，高分子通报，2016，(9), 45.)

[25] Pickering, S. U. J. Am. Chem. Soc. 1907, 91, 2001.

[26] Binks, B. P. Curr. Opin. Colloid Interface Sci. 2002, 7, 21

[27] Lin, Y.; Skaff, H.; Emrick, T.; Dinsmore, A. D.; Russell, T. P. Science 2003, 299, 226.

[28] Melle, S.; Lask, M.; Fuller, G. G. Langmuir 2005, 21, 2158.

[29] Komazaki, Y.; Hirama, H.; Torii, T. J. Appl. Phys. 2015, 117, 154506.

[30] Binks, B. P.; Lumsdon, S. O. Langmuir 2000, 16, 2539.

[31] Liang, F. X.; Zhang, C. L.; Yang, Z. Z. Adv. Mater. 2014, 26, 6944.

[32] Kline, T. R.; Paxton, W. F.; Mallouk, T. E.; Sen, A. Angew. Chem., Int. Ed. 2005, 44, 744.

[33] Laocharoensuk, R.; Burdick, J.; Wang, J. ACS Nano 2008, 2, 1069.
[34] Wang, J.; Manesh, K. M. Small 2010, 6, 338.

[35] Gao, W.; Uygun, A.; Wang, J. J. Am. Chem. Soc. 2012, 134, 897.

[36] Jonathan, R. H.; Richard, A. L. J.; Anthony, J. R.; Tim, G.; Reza, V.; Ramin, G. Phys. Rev. Lett. 2007, 99, 048102-1.

[37] Ge, Y. E.; Wang, T.; Zheng, M. F.; Jiang, Z. Z.; Wang, S. Nanotechnology 2019, 30, 315702 .

[38] Zheng, J.; Wang, J. G.; Xiong, Z.; Wan, Z. H.; Zhan, X. J.; Yang, S. J.; Chen, J. W.; Dai, J.; Tang, J. Y. Adv. Funct. Mater. 2019, 29, 1901768.

[39] Xuan, M. J.; Wu, Z. G.; Shao, J. X.; Dai, L. R.; Si, T. Y.; He, Q. J. Am. Chem. Soc. 2016, 138, 6492.

[40] Wu, Y. J.; Wu, Z. G.; Lin, X. K.; He, Q.; Li, J. B. ACS Nano 2012, 6, 10910.

[41] Crossley, S.; Faria, J.; Shen, M.; Resasco, D. E. Science 2010, 327, 68.

[42] Kirillova, A.; Schliebe, C.; Stoychev, G.; Jakob, A.; Lang, H.; Synytska, A. ACS Appl. Mater. Interfaces 2015, 7, 21218.

[43] Wang, C.; Yin, H.; Dai, S.; Sun, S. Chem. Mater. 2010, 22, 3277.

[44] Valadares, L. F.; Tao, Y. G.; Zacharia, N. S.; Kitaev, V.; Galembeck, F.; Kapral, R.; Ozin, G. A. Small 2010, 6, 565.

[45] Liu, Y. J.; Hu, J. K.; Yu, X. T.; Xu, X. Y.; Gao, Y.; Li, H. M.; Liang, F. X. J. Colloid Interface Sci. 2017, 490, 357.

[46] Xu, X. Q.; Deng, C. H.; Gao, M. X.; Yu, W. J.; Yang, P. Y.; Zhang, X. M. Adv. Mater. 2006, 18, 3289 . 